\title{
Design and Development of Electrostatically Driven Uniaxial Tensile Test Device for Silicon Nanowires
}

\author{
Tatsuya Fujii, ${ }^{1,2^{*}}$ Koichi Sudoh, ${ }^{3}$ Shozo Inoue, ${ }^{1}$ and Takahiro Namazu ${ }^{1}$ \\ ${ }^{1}$ Department of Mechanical Engineering, University of Hyogo, 2167 Shosha, Himeji, Hyogo 671-2201, Japan \\ ${ }^{2}$ JSPS Research Fellow, Japan Society for the Promotion of Science, \\ 5-3-1 Kojimachi, Chiyoda-ku, Tokyo 102-0083, Japan \\ ${ }^{3}$ The Institute of Scientific and Industrial Research, Osaka University, \\ 8-1 Mihogaoka, Ibaraki, Osaka 567-0047, Japan
}

(Received November 18, 2015; accepted December 21, 2015)

Keywords: silicon nanowire, tensile test, electrostatic actuator, capacitive sensor, mechanical property, MEMS

In this paper, the design and development of an electrostatically actuated microelectromechanical systems (MEMS) device for the tensile test of Si nanowires (NWs) are described. The device is composed of a comb-drive electrostatic actuator for generating uniaxial tensile force, capacitive sensors for measuring tensile force and displacement, an electrothermal actuator with a ratchet for clamping and releasing a sample stage, and a force calibration mechanism. The resolution of the tensile elongation measurement is $1 \mathrm{~nm}$, determined by a resolution of $0.1 \mathrm{fF}$ on an LCR meter. The tensile force is derived from the displacement and the spring constant of the support beams of the driven sensor. The theoretical resolution of tensile force measurements ranges from 10 to $263 \mathrm{nN}$, depending on the stiffness of the specimen. Electrical insulating structures for minimizing electrical signal noise are designed to accurately measure the capacitance change of the two capacitive sensors. To demonstrate these possibilities, Si NWs fabricated using a focused ion beam (FIB) were characterized using a scanning electron microscope (SEM). The Young's modulus and fracture strength were 127.7 and $5.4 \mathrm{GPa}$, respectively, indicating that FIB damage affects these mechanical characteristics.

\section{Introduction}

Because of the rapid progress of nano-scale fabrication technologies, nano-electromechanical systems (NEMS) have been studied for a variety of industrial applications..$^{(1-5)}$ Nano-sized materials such as nanowires (NWs) and nanotubes play important roles as mechanical and electrical components in NEMS.(4-9) To improve the performance and reliability of NEMS, the mechanical characteristics of these nanomaterials must be experimentally examined and the results reflected in the design of improved NEMS. Experimentally evaluating the mechanical properties of nanomaterials is rare compared to evaluations of electrical properties because there are many technical difficulties in chucking nano-specimens and measuring force and displacement during the experiment. Even if the specimen size is on the nanometer scale, the uniaxial tensile test is the most popular method, just as it is with bulk specimens because the stress-strain relation of a specimen

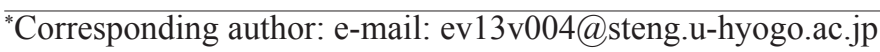


can be directly obtained. ${ }^{(10-13)}$ Accurately obtaining this relation leads to accurate derivation of mechanical characteristics, such as Young's modulus, yield strength, and fracture strength, which are necessary for the mechanical design.

In this report, we focus on the design and development of a tensile testing device based on micro-electromechanical systems (MEMS) technology for Si NWs. The design concept and idea to achieve accurate nanoscale tensile testing of Si NWs are described. The performance of the devices produced are presented. Si NWs fabricated using a focused ion beam (FIB) were put under tension to determine the accuracies of force and displacement measurements and the abilities of the device.

\section{Device Design and Fabrication}

\subsection{Concept}

Figure 1(a) shows a schematic of the specially designed tensile test device for Si NWs. The device consists of a comb-drive electrostatic actuator for uniaxial tensile force, an actuator-side capacitive sensor (act sensor) for displacement measurement, a driven capacitive sensor (driven sensor) for displacement and force measurement, and a sample stage. The entire comb structure is supported by U-shaped suspension beams, ${ }^{(14)}$ which can freely move in the in-plane tensile direction only. The sample stage is located between the two sensors. A NW specimen is made directly on the stage like a bridge using the FIB system's probe manipulation and film deposition functions reported elsewhere. ${ }^{(15-17)}$ The device includes three insulators to realize several different electrical functions during the test. These structures enable us to accurately measure the capacitance change of two sensors because the capacitance sensing is perfectly decoupled from the actuation voltage signal. ${ }^{(18,19)}$

Figure 1(b) illustrates the relationship between applied voltage and displacement. The solid and dashed lines are indicative of the displacement of the act and driven sensors, respectively. The displacement $d_{\mathrm{s}}$ of a specimen can be measured by subtracting the displacement $d_{\text {driven }}$ of the driven sensor from the displacement $d_{\text {act }}$ of the act sensor, which is expressed as

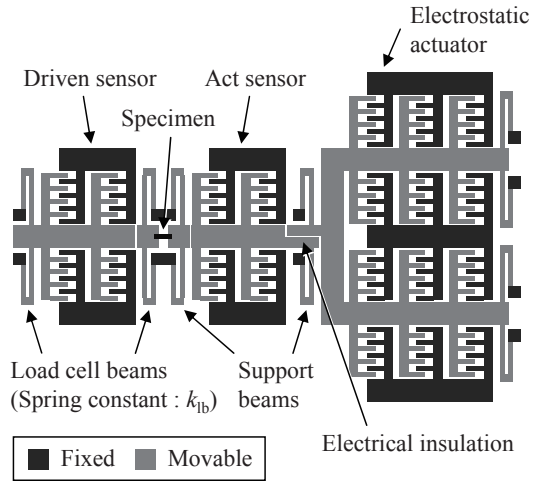

(a)

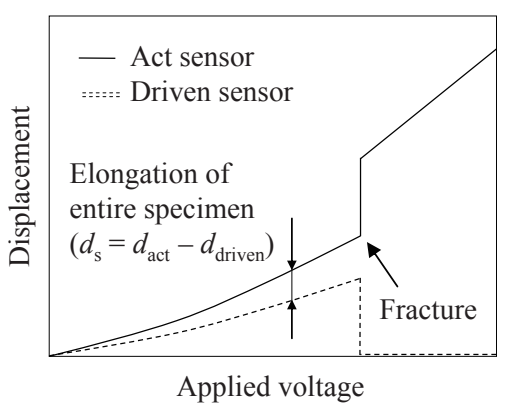

(b)

Fig. 1. (a) Schematic of the specially designed MEMS tensile testing device for Si NWs. (b) Illustration of the relationship between applied voltage to the actuator and displacements of two capacitive sensors. 


$$
d_{\mathrm{s}}=d_{\mathrm{act}}-d_{\mathrm{driven}} \text {. }
$$

The tensile force $F_{\mathrm{s}}$ applied to a specimen is calculated by multiplying the displacement $d_{\text {driven }}$ by the spring constant $k_{\mathrm{lb}}$ of the support beams (load cell beams) for the driven sensor, which is given by

$$
F_{\mathrm{s}}=k_{\mathrm{lb}} d_{\text {driven }}
$$

To measure the $F_{\mathrm{s}}$, both the $k_{\mathrm{lb}}$ and the $d_{\text {driven }}$ must be derived accurately.

\subsection{Fundamental design}

It is ideal to apply a large tensile force to a specimen at a low voltage $(<60 \mathrm{~V})$. Here, the maximum drive voltage was set on the basis of Paschen's law describing the breakdown voltage $V_{\text {break }}$ between parallel electrodes. ${ }^{(20)}$ The $V_{\text {break }}$ is expressed as a function of the gas pressure $p$ and the gap distance $g$,

$$
V_{\text {break }}=\frac{B p g}{\ln \frac{A p g}{\ln (1+1 / \gamma)}}=f(p g) \text {, }
$$

where $A$ and $B$ are the gas-dependent constants, and $\gamma$ is the secondary electron emission coefficient of the electrode materials. In the case of constant $p$, Eq. (3) indicates that $V_{\text {break }}$ can be directly estimated from only $g$, as defined in Fig. 2(a). Figure 2(b) shows the Paschen curve for $A=10.95$ $\mathrm{m}^{-1} \mathrm{~Pa}^{-1}, B=273.78 \mathrm{Vm}^{-1} \mathrm{~Pa}^{-1}$ (air), $\gamma=0.73$ (Si electrode), and $p=1$ atm. ${ }^{(21)}$ When $g$ is larger than $2.5 \mu \mathrm{m}$, the relation between $g$ and $V_{\text {break }}$ is almost linear. In this study, the $g$ was set at $4 \mu \mathrm{m}$ in consideration of the yield percentage of device fabrication by deep reactive ion etching (DRIE). (As the device thickness $t$ is $35 \mu \mathrm{m}$, the aspect ratio $t / g$ is 8.75.) Thus, $V_{\text {break }}$ is estimated to be $67.8 \mathrm{~V}$ for a $4 \mu \mathrm{m}$ gap.

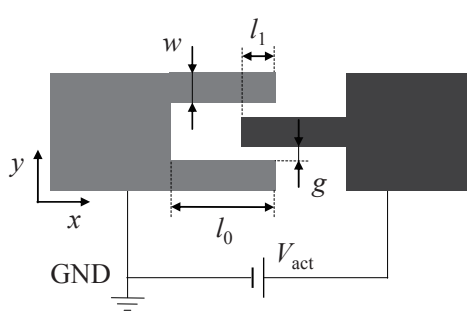

(a)

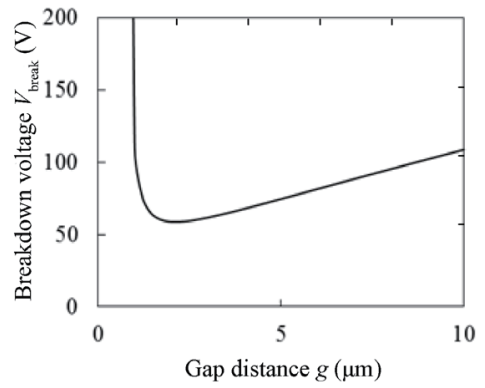

(b)

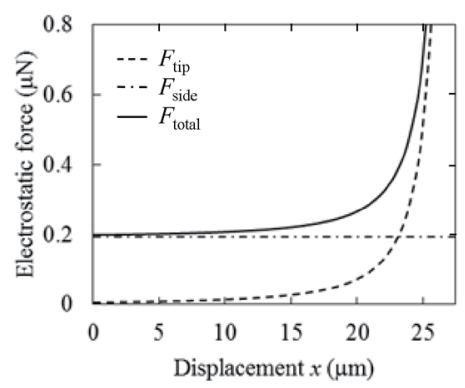

(c)

Fig. 2. The design of an electrostatic actuator. (a) Two-dimensional model of one comb finger. (b) Paschen curve for Si electrodes in air as a function of gap distance. (c) The relationship between displacement and electrostatic force of the actuator. 
Preventing a pull-in phenomenon is an important design consideration. The electrostatic force $F_{\text {total }}$ of the entire comb-drive actuator is expressed as

$$
F_{\text {total }}=n_{\text {act }} \varepsilon_{0} t V_{\text {act }}^{2}\left\{\frac{w}{\left(l_{0}-l_{1}-x\right)^{2}}+\frac{1}{g}\right\}=F_{\text {tip }}+F_{\text {side }},
$$

where $n_{\text {act }}$ is the number of comb structures, $\varepsilon_{0}$ is the dielectric constant in vacuum, $V_{\text {act }}$ is the applied voltage to the actuator, $w$ is the comb finger width, $l_{0}$ and $l_{1}$ are the comb finger length and overlapped length, and $x$ is the displacement in the $x$ direction (= tensile direction) of the actuator. The first and second terms indicate the $x$ directional electrostatic forces in the comb-tip $F_{\text {tip }}$ and comb-side $F_{\text {side, }}$, respectively. Figure 2(c) shows the relationship between displacement $x$ and electrostatic force for $F_{\text {tip }}$ and $F_{\text {side. }}$ Here, the other parameters are set as $V_{\text {act }}=40 \mathrm{~V}, w=6 \mu \mathrm{m}, l_{0}$ $=35 \mu \mathrm{m}$, and $l_{1}=7 \mu \mathrm{m}$. The dashed, one-dot chain, and solid lines indicate the $F_{\text {tip }}, F_{\text {side, }}$, and $F_{\text {total }}$, respectively. The $F_{\text {tip }}$ gradually increases as $x$ increases to $20 \mu \mathrm{m}$. Above $20 \mu \mathrm{m}$, however, the $F_{\text {tip }}$ rapidly increases due to the front pull-in phenomenon of the actuator, which must be avoided for quasi-static tensile loading. In our design, the maximum stroke of the actuator was set to $14 \mu \mathrm{m}$ to achieve smooth tensile loading. ${ }^{(22)}$

In the design of capacitive sensors, it is important to achieve high resolution, linearity, and stability during the test. The capacitance change $\Delta C$ is expressed as

$$
\Delta C=\frac{2 n_{\mathrm{sens}} \varepsilon_{0} t}{g} d
$$

where $n_{\text {sens }}$ and $d$ are the number and the displacement of comb structures, respectively. The value of $\Delta C$ does not depend on the displacement $x$, and it can directly provide $d$.

Using Eqs. (1)-(5), three types of MEMS tensile test devices were designed. Table 1 lists the dimensions and calculated performance of the devices. We set the minimum measurement

Table 1

The design parameters for main electromechanical parts of the devices.

\begin{tabular}{lcccc}
\hline Parameters & & Type A & Type B & Type C \\
\hline $\begin{array}{l}\text { Device thickness }(\mu \mathrm{m}) \\
\text { Comb structures }\end{array}$ & $t$ & 35 & 35 & 35 \\
$\quad$ Comb gap $(\mu \mathrm{m})$ & $g$ & & & 4 \\
Electrostatic actuator & & 4 & 4 & \\
$\quad n_{\text {act }}$ & 1024 & 2048 & 3072 \\
$\quad$ Number of combs & $V_{\text {act }}$ & $\leq 60$ & $\leq 60$ & $\leq 60$ \\
$\quad$ Drive voltage $(\mathrm{V})$ & & & & \\
Actuator-side capacitive sensor & $n_{\text {sens }}$ & 448 & 448 & 448 \\
$\quad$ Number of combs & & 0.07 & 0.07 & 0.07 \\
$\quad$ Sensor sensitively (fF/nm) & & & & \\
Driven capacitive sensor & $n_{\text {sens }}$ & 640 & 640 & 640 \\
$\quad$ Number of combs & & 0.10 & 0.10 & 0.10 \\
$\quad$ Sensor sensitively (fF/nm) & $k_{\mathrm{lb}}$ & 10.1 & 67.2 & 263.4 \\
Stiffness of load cell beams $(\mathrm{N} / \mathrm{m})$ & $k_{\mathrm{sb}}$ & 33.8 & 44.1 & 54.3 \\
Stiffness of support beams $(\mathrm{N} / \mathrm{m})$ & & & & \\
\hline
\end{tabular}


resolution of specimen elongation at $1 \mathrm{~nm}$ as the common specification for all devices. The stiffness $k_{\mathrm{lb}}$ of the load cell beams and the number of combs $n_{\text {act }}$ of the actuator were changed in consideration of various specimens having different material properties and dimensions. Si NWs with cross-sectional dimensions from 10, 50, and $100 \mathrm{~nm}^{2}$ to 50,100 , and $200 \mathrm{~nm}^{2}$ were considered for the design of the Type A, B, and $\mathrm{C}$ devices, respectively. These dimensional ranges were determined under the condition that a tensile strain of $10 \%$ was applied to the respective NWs at $60 \mathrm{~V}$. When the device with stiffness $k_{\mathrm{lb}}$ less than or equal to the specimen's stiffness was used for tensile testing, the displacement $d_{\text {driven }}$ of the driven sensor was equal to or larger than $1 \mathrm{~nm}$ for a specimen elongation of $1 \mathrm{~nm}$. Therefore, the capacitance change $\Delta C_{\text {driven }}$ of the driven sensor is equal to or greater than $0.1 \mathrm{fF}$. Assuming that the measurement displacement resolution was $1 \mathrm{~nm}$, the stiffness $k_{\mathrm{lb}}$ of the load cell beam for the driven sensor was set to 10 (Type A), 67 (Type B), and $263 \mathrm{~N} / \mathrm{m}$ (Type C), which correspond to a force measurement resolution of 10,67 , and $263 \mathrm{nN}$, respectively.

\subsection{Stopper mechanism for restraining sample vibration}

The MEMS device designed includes a stopper mechanism for restraining vibration during specimen test setup. The mechanism is located at both sides of the sample stage. Figure 3(a) shows a schematic of the stopper on one side. This device consists of two ratchet structures for fixing the sample stage, a V-shaped electrothermal actuator (ETA) for releasing the stage fixation,

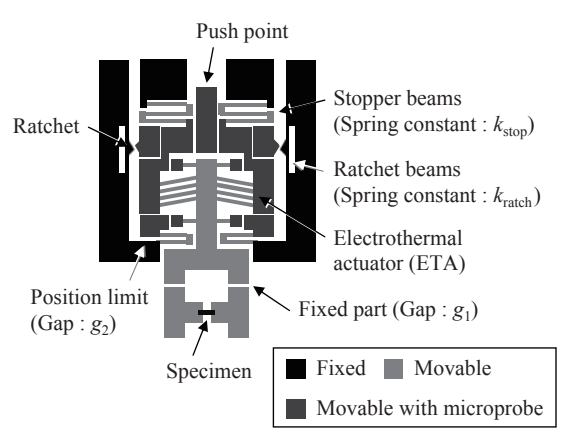

(a)

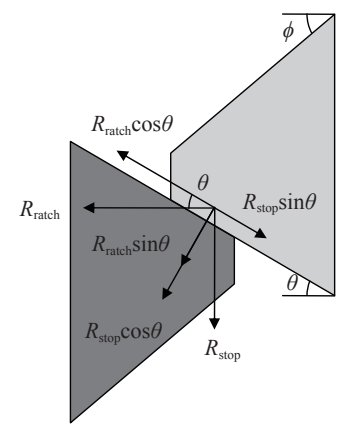

(c)

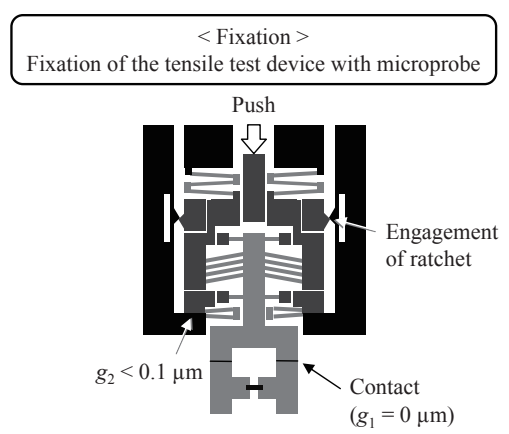

(b)

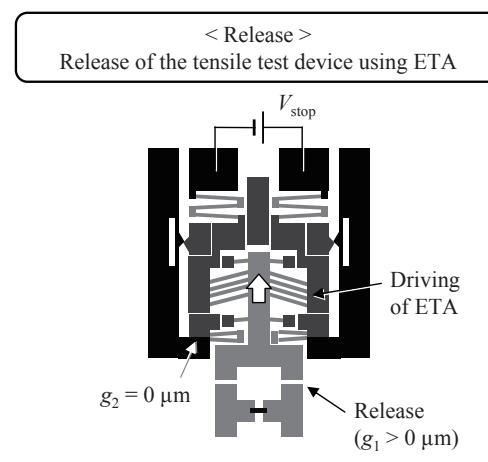

(d)

Fig. 3. (a) Schematic of the stopper mechanism for minimizing sample vibration. (b) The sample stage fixation procedure. (c) The mechanical dynamics when the ratchet is engaged. (d) The sample stage release procedure. 
and a frame. A movable structure including the ratchet and ETA is supported by four U-shaped suspension beams. The structure can move only $21.0 \mu \mathrm{m}$ corresponding to the gap $g_{1}$. The value of $g_{1}$ is smaller by approximately $0.1 \mu \mathrm{m}$ than the gap $g_{2}$ corresponding to the spacing between suspension beam structures. As shown in Fig. 3(b), first the movable structure including the ETA is moved with a micro probe to clamp the sample stage, which is always fastened using the ratchet. Then a NW specimen is fabricated using FIB and is mounted on the sample stage using a micro probe in the FIB equipment. After that, the MEMS device with a NW sample is transferred to a scanning electron microscope (SEM). Then a bias voltage of $8 \mathrm{~V}$ is applied using a direct-current (DC) power supply to the ETA to release the stopper from the sample stage.

To clamp the stage as completely as possible, the configuration of the ratchet is very important. As shown in Fig. 3(c), when staying in balance, the mechanics of a ratchet can be expressed as

$$
\begin{gathered}
R_{\text {ratch }}=\frac{R_{\text {stop }}(\sin \theta+\mu \cos \theta)}{\cos \theta-\mu \sin \theta}=R_{\text {stop }} \tan (\theta+\rho), \\
\text { if } \tan (\theta+\rho) \leq 0, R_{\text {ratch }}=R_{\text {stop }} \tan (\theta+\rho) \leq 0, \\
\text { therefore, } \tan (\theta+\rho)>0,
\end{gathered}
$$

where $R_{\text {ratch }}$ and $R_{\text {stop }}$ are the reaction forces at the boundary, $\theta$ is the angle between the contact surface and the transversal to the direction of motion, $\mu$ is the static friction coefficient, and $\rho$ is the angle of friction $(\mu=\tan \rho)$. In the DRIE-fabricated surface, the value of $\mu$ is known to be approximately $0.65 .^{(23)}$ The boundary condition for the ratchet, which is not released by its own reaction force (self-locking), is $\theta<57.0^{\circ}$, so the angle $\theta$ after the ratchet is engaged was set to $45^{\circ}$. On the other hand, the angle $\phi$ before the ratchet is engaged was set to $60^{\circ}$ to reduce the engaging force.

To release the stopper before the test, optimizing the design of the ETA is required. The stiffness $k_{y}$ in the $y$ direction of the ETA shown in Fig. 4(a) is given by

$$
k_{y}=2 n_{\mathrm{hb}}\left(\sin ^{2} \psi \frac{E w_{\mathrm{hb}} t}{l_{\mathrm{hb}}}+\cos ^{2} \psi \frac{E w_{\mathrm{hb}}^{3} t}{l_{\mathrm{hb}}^{3}}\right)+2 n_{\mathrm{sb}} \frac{E w_{\mathrm{sb}}^{3} t}{l_{\mathrm{sb}}^{3}}
$$

where $n_{\mathrm{hb}}$ is the number of V-shaped heater beams, $\psi$ is the angle between the heater beam and the transverse direction, $E$ is the Young's modulus of the ETA material, and $t, w_{\mathrm{hb}}$, and $l_{\mathrm{hb}}$ are the thickness, width, and length of the heater beam, respectively. ${ }^{(24)}$ The term $n_{\mathrm{sb}}$ is the number of support beams, and $w_{\mathrm{sb}}$ and $l_{\mathrm{sb}}$ are the width and length of the support beams, respectively. The force $F_{\mathrm{y}}$ in the $y$ direction generated by thermal expansion of the ETA is given by

$$
F_{y}=2 n_{\mathrm{hb}} E A \alpha \Delta T \sin \psi,
$$

where $\alpha$ is the thermal expansion coefficient of the heater beam, and $A$ and $\Delta T$ are the crosssectional area and temperature increase of the heater beam, respectively. The displacement $d_{\mathrm{y}}$ of the ETA in the $y$ direction is expressed as the following equation, which is derived from Eqs. (7) and (8)

$$
d_{y}=F_{y} / k_{y}
$$




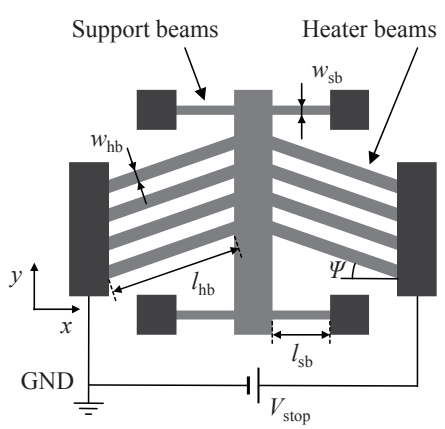

(a)

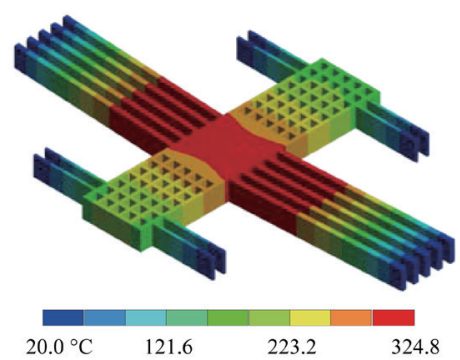

(c)

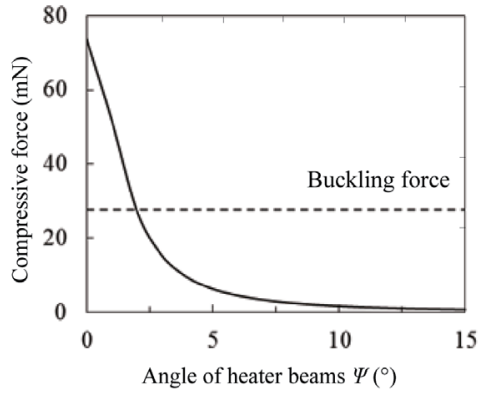

(b)

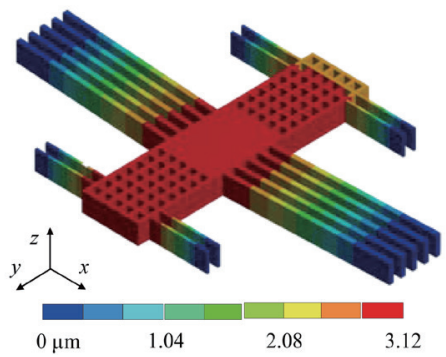

(d)

Fig. 4. (Color online) The design of an ETA. (a) Two-dimensional model of an ETA. (b) The relationship between beam angle and compressive force originating from the thermal expansion. (c) The temperature and (d) $y$-direction displacement distributions in the optimized ETA.

From Eq. (9), when the stiffness of the support beams is smaller than that of the heater beams, the displacement of the ETA depends on the magnitude of the temperature increase $\Delta T$, the heater beam length $l_{\mathrm{hb}}$, and the beam angle $\psi$. In this study, the maximum temperature in the ETA was restricted to be below $300{ }^{\circ} \mathrm{C}$, which is around half of the brittle-ductile transition temperature for bulk $\mathrm{Si}$. Therefore, the structures of the ETA do not deform plastically. ${ }^{(13,25)}$ In the case of the same temperature increase, as the beam length $l_{\mathrm{hb}}$ elongated, the displacement $d_{\mathrm{y}}$ of the ETA increased. However, longer beams cause a decrease in the buckling force $P_{\text {buck }}$, which can be expressed as a function of the minimum moment of inertia $I_{\min }$

$$
P_{\text {buck }}=\frac{\pi^{2} E I_{\min }}{l_{\mathrm{hb}}^{2}}=\frac{\pi^{2} E w_{\mathrm{hb}}^{3} t}{12 l_{\mathrm{hb}}^{2}} .
$$

In our device, a beam length of $300 \mu \mathrm{m}$ was chosen in consideration of the buckling force and the size of the entire ETA. In the $8 \mu \mathrm{m}$-wide and $35 \mu \mathrm{m}$-thick heater beam, a buckling force of 27.7 $\mathrm{mN}$ can be produced in the normal direction of the substrate. Figure 4(b) shows the relationship between the beam angle and the compressive force originating from the thermal expansion of a single heater beam at $\Delta T=300{ }^{\circ} \mathrm{C}$. The ETA beam buckles when the angle is smaller than approximately $2^{\circ}$; hence, an angle of $3^{\circ}$ was chosen. Figures $4(\mathrm{c})$ and $4(\mathrm{~d})$ show temperature and $y$-direction displacement distributions in the optimized ETA, respectively. The ETA consists of five pairs of V-shaped heater beams $300-\mu \mathrm{m}$ long, $8-\mu \mathrm{m}$ wide, and $35-\mu \mathrm{m}$ thick, and two pairs of 
support beams $110-\mu \mathrm{m}$ long and $4-\mu \mathrm{m}$ wide. Commercial finite element analysis (FEA) software, ANSYS Workbench, was used. The inputs of mechanical and electrical properties for the Si that is a structural material for the ETAs are Young's modulus $E=168.9 \mathrm{GPa}$, Poisson's ratio $v=$ 0.064 , resistivity $\rho=0.02 \Omega \mathrm{cm}$, thermal conductivity $\lambda=34 \mathrm{Wm}^{-1} \mathrm{~K}^{-1}$, and a thermal expansion coefficient $\alpha=2.5 \times 10^{-6} \mathrm{~K}^{-1}$.(24) The actuation voltage of $8 \mathrm{~V}$ in a vacuum was applied to the FEA in consideration of in situ SEM actuation. The highest temperature, $324.8^{\circ} \mathrm{C}$, was obtained in the center of the ETA. The maximum displacement at this temperature was $3.12 \mu \mathrm{m}$, which is sufficient to release the stopper; therefore, the designed ETA can be used as a stopper mechanism for the sample holder.

\subsection{Fabrication}

Figure 5 shows the fabrication process for the device. A p-type SOI wafer with a $35-\mu \mathrm{m}$-thick device layer of $0.02 \Omega \mathrm{cm}, 2-\mu \mathrm{m}$-thick buried oxide (BOX) layer, and $200-\mu \mathrm{m}$-thick handle layer was chosen as a starting material [Fig. 5(a)]. First, 30-nm-thick $\mathrm{Cr}$ and 300-nm-thick Au thin films were deposited on the top surface by sputtering, and the electrode for wire bonding was formed [Fig. 5(b)]. Then, 100-nm-thick Al thin film was coated on the top surface by sputtering, and the actuator and sensor pattern were formed on the surface. The backside surface was then patterned with 200-nm-thick Al thin film for a DRIE mask [Fig. 5(c)]. After that, both surfaces of the wafer were etched by DRIE with a BOSCH process recipe [Fig. 5(d)]. The remaining Al layers were removed [Fig. 5(e)]. The exposed BOX layer was finally removed using hydrofluoric (HF) vapor etching to diminish sticking [Fig. 5(f)]. ${ }^{(26)}$

SEM images of the MEMS device produced with 2048 pairs of comb-drive actuators are shown in Fig. 6. The device could be precisely fabricated with the conventional micro-fabrication technologies described. The sample stage, comb structure, and ratchet structure, which are the main mechanical components for the nanoscale tensile test, were finely formed. The sidewalls had a scallop pattern with $1 \mu \mathrm{m}$ pitch and were finely etched by DRIE.

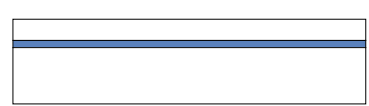

(a) SOI wafer

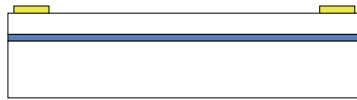

(b) $\mathrm{Au} / \mathrm{Cr}$ patterning

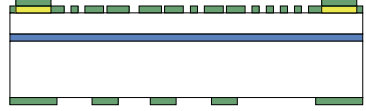

(c) Al patterning

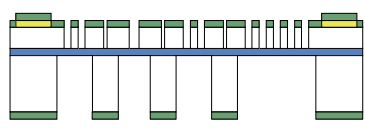

(d) DRIE

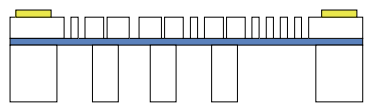

(e) Al removal

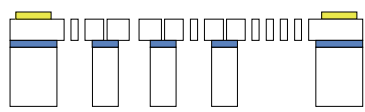

(f) Vapor HF etching

$$
\square \mathrm{Si} \square \mathrm{SiO}_{2} \quad \square \mathrm{Au} / \mathrm{Cr} \quad \square \mathrm{Al}
$$

Fig. 5. (Color online) Fabrication process for the device. (a) SOI wafer with $35-\mu \mathrm{m}$-thick device layer. (b) Patterning of $\mathrm{Au} / \mathrm{Cr}$ layers. (c) Patterning of Al layers on both surfaces. (d) DRIE process. (e) Removal of Al layers. (f) $\mathrm{HF}$ acid vapor etching of the sacrificed layer. 


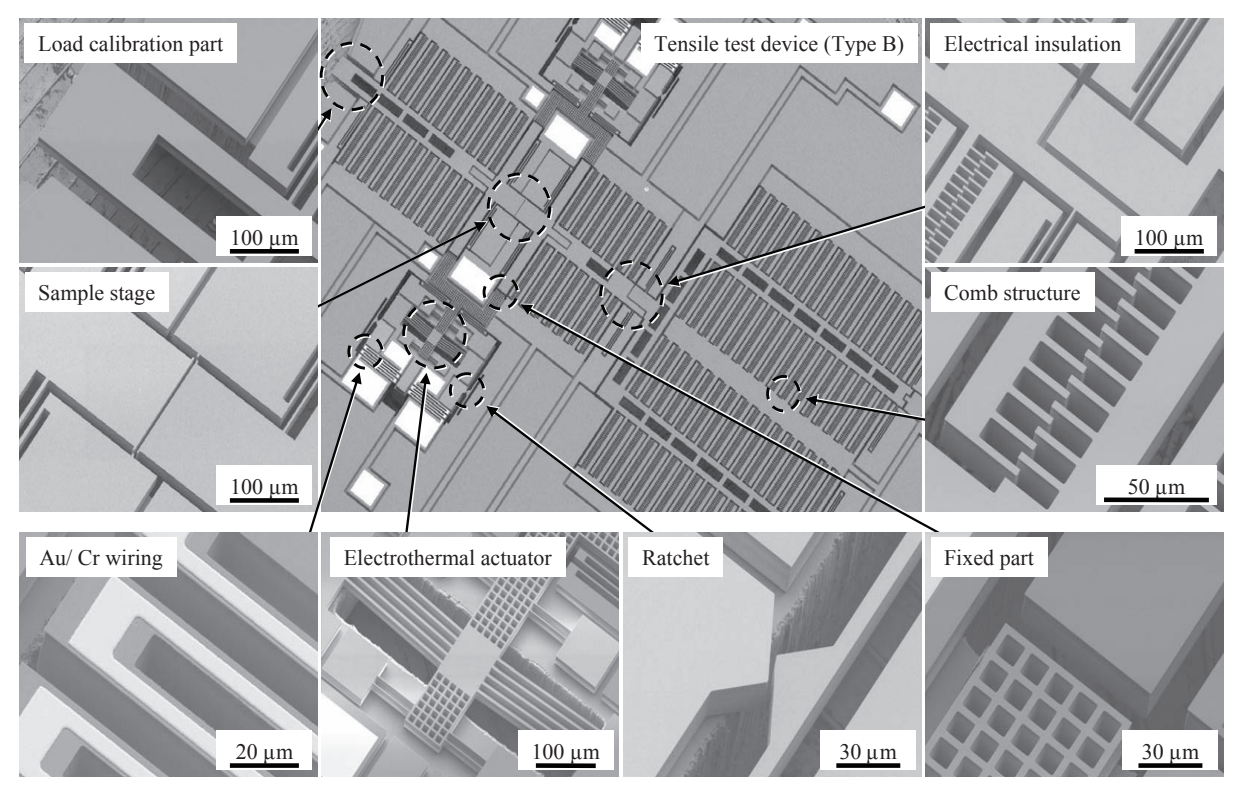

Fig. 6. SEM images of the MEMS device produced with 2048 pairs of comb-drive actuators.

\section{In Situ SEM Tensile Testing System}

Figure 7 illustrates a nanoscale tensile test system in a SEM. A bias voltage is applied using a data acquisition system (DAQ: National Instrument Corp., USB-6211) and a direct current amplifier (NF Corp., BA4825) to the comb drive actuator. In the circuit between the DC amplifier and the actuator, an electrical resistance of $1 \mathrm{M} \Omega$ is integrated to avoid a short circuit. The output voltage is measured using a computer via an analog input (AI) terminal of the DAQ. The displacements of the act and driven sensors are measured using two high-precision $(\sigma<1 \mathrm{fF})$ LCR meters (Keysight Technologies Inc., E4980A). Ideally, the resolution of a displacement measurement is less than $1 \mathrm{~nm}$, depending on electrical noise level and temperature fluctuation. In this study, we used a capacitive sensor as well as a digital image analysis system for finer measurements. The image analysis system (resolution with 1/2 sized display: $640 \times 512$ pixels, frame rate 60 frames/s in a grayscale image) in a SEM (Hitachi High-Technologies Corp., S-4800) can measure relative displacement between the two memorized marks in an image. The SEM images obtained are fed directly into a computer at 10 frames/s using a DVI capture unit (Epiphan System Inc., DVI2USB3.0). When an observation area is $10 \mu \mathrm{m}$ based on the length of a NW $(5 \mu \mathrm{m})$, the resolution is theoretically calculated to be $16 \mathrm{~nm} /$ pixel.

\section{Experimental Results}

\subsection{Device performance}

Figure 8(a) shows representative results of displacement measurements using the image analysis system. In this evaluation, the Type B device was used. The dashed and solid lines indicate the voltage-displacement relations estimated using designed dimensions and actual dimensions, 


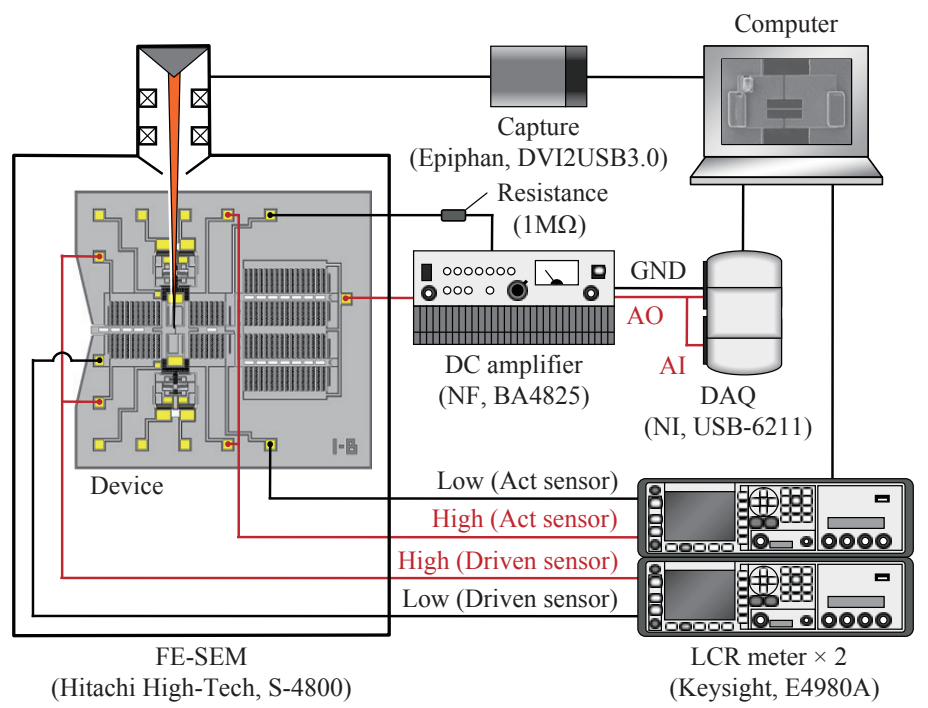

Fig. 7. (Color online) Illustration of the in situ nanoscale tensile test system in a SEM with the displacement measurement system by digital image analysis.

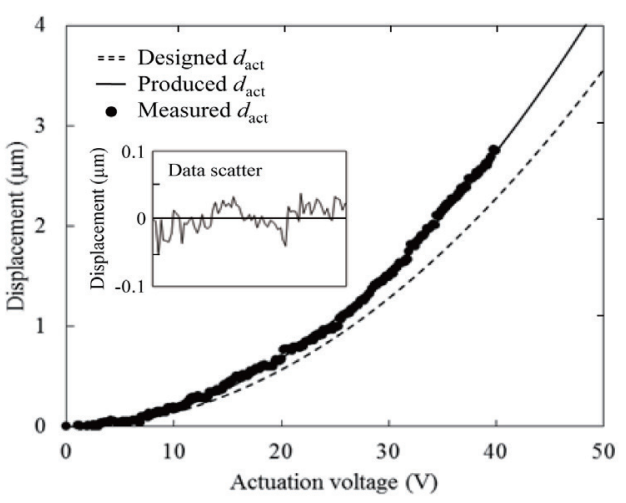

(a)

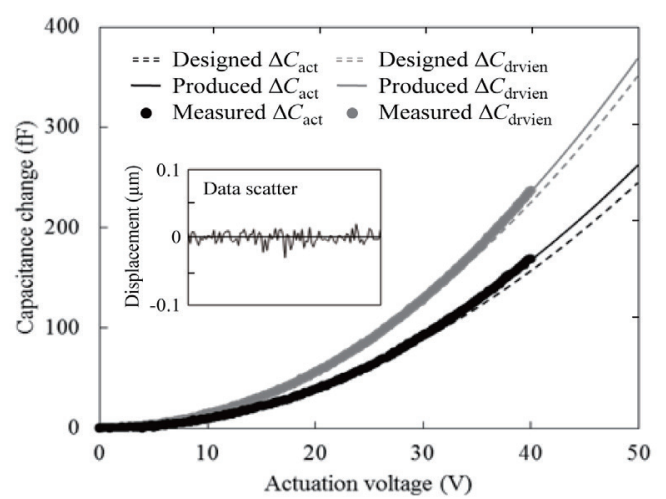

(b)

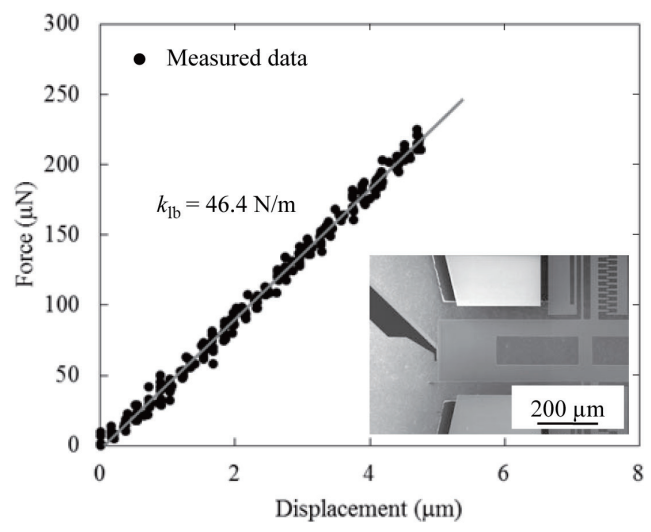

(c)

Fig. 8. Representative results of (a) displacement measurement using the image analysis system, (b) displacement measurement of two capacitive sensors using an LCR meter, and (c) force measurement. 
respectively, and the solid circles indicate the experimental data. The voltage-displacement relation estimated using designed dimensions has a quadric shape because the displacement increases with the square of applied voltage $V_{\text {act }}$, as shown in Eq. (4). The relation estimated using actual dimensions differs from that estimated using designed dimensions. At the same voltage, the displacement in actual dimensions is approximately 1.2 times larger than that in the designed dimensions. This indicates that, compared with the designed device, the actual device possesses either a small beam width or large gap between the movable and fixed combs transverse to the tensile direction. The measured relation is plotted based on the relation estimated using actual dimensions, which implies that the displacement of the act sensor can be estimated closely from the applied voltage and actual dimensions. As shown in the small graph, the range of data scatter is within $\pm 30 \mathrm{~nm}$. The cause of the scatter may be related to a defocusing of the structural edge.

Figure 8(b) shows representative results of displacement measurements using two capacitive sensors. The difference in black and gray plots indicates the difference in the act and driven sensors. The dashed and solid lines indicate the change in the voltage-capacitance relations estimated using designed dimensions and actual dimensions, respectively. These plots show the experimental data using an LCR meter. The voltage-capacitance relation estimated using designed and actual dimensions has a quadric shape as does the voltage-displacement relation. For each sensor, the measured data are plotted based on the relations estimated using actual dimensions. The sensitivities of the act and driven sensors are calculated to be 0.059 and $0.083 \mathrm{fF} / \mathrm{nm}$, respectively, which are almost the same as the estimated values using the actual dimensions. The range of the data scatter is within $\pm 10 \mathrm{~nm}$, which is one-third of that in the image analysis. Reducing electronic noise is essential for high measurement accuracy.

Figure $8(\mathrm{c})$ shows representative results of force measurements. The force data were measured using the micro-force sensing probe (FemtoTools AG, FT-S1000-LAT, Resolution: $0.05 \mu \mathrm{N}$ ) mounted on the nano manipulation stage (Sanyu Co., Ltd., NMS T14003) in a SEM. Using the probe, a displacement of $5 \mu \mathrm{m}$ was applied to the driven sensor, as shown in the SEM image in the graph. The displacement-force relation is linear, although some data spread is seen due to digital image analysis. The spring constant was $46.4 \mathrm{~N} / \mathrm{m}$, which is approximately $1.5 \%$ smaller than the value estimated using actual dimensions. This difference may be caused by the scallops on the sidewalls.

Figure 9(a) shows the SEM images of the stopper before and after engaging the ratchet. Before engaging, the initial gaps $g_{1}$ and $g_{2}$ were 21.0 and $21.1 \mu \mathrm{m}$, respectively. After engaging, the stopper could contact with and clamp the sample stage. By applying a voltage of $8 \mathrm{~V}$ to the ETA, as shown in Figure 9(b), a small gap of $3.2 \mu \mathrm{m}$ in the $g_{1}$ portion could be generated. The gap value was in very good agreement with the FEA result shown in Fig. 4(d). Gap generation means that the sample stage can be completely released from the stopper before the test. Compared with our previous devices without this mechanism, ${ }^{(15,16)}$ the yield rate in placing NW specimens was improved approximately 5 -fold.

\subsection{Tensile testing of FIB-fabricated Si NWs}

For the evaluation of the MEMS device developed, the tensile test of Si NWs was performed using the Type B device in an SEM. Si NWs made from silicon-on-nothing (SON) membranes were individually mounted on the device using probe manipulation and film deposition functions 


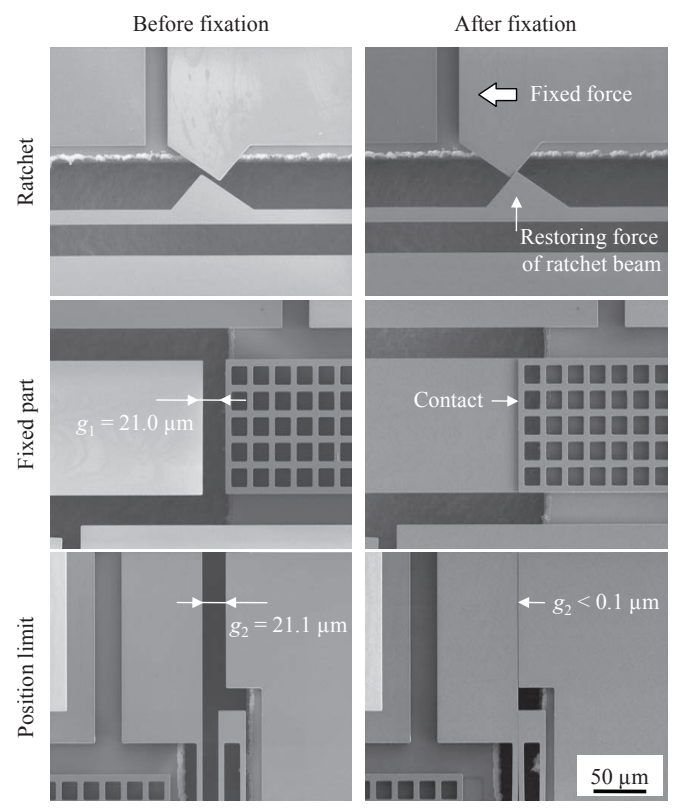

(a)

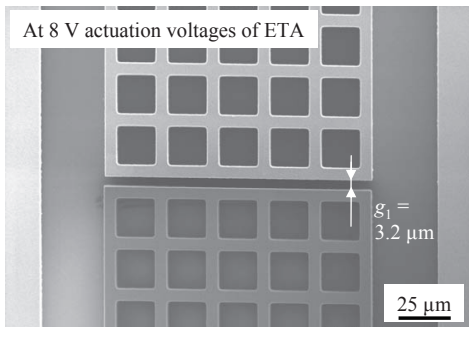

(b)

Fig. 9. SEM images of (a) the main portions of stopper device before and after engaging the ratchet, and (b) the release of the stopper from the sample stage by applying a voltage of $8 \mathrm{~V}$ to the ETA.

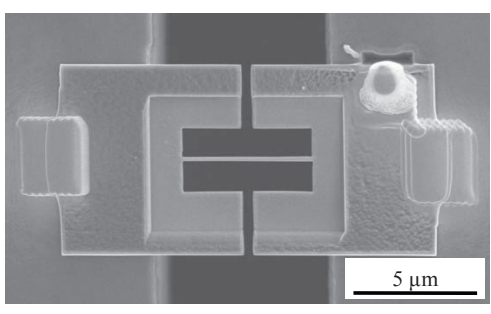

(a)

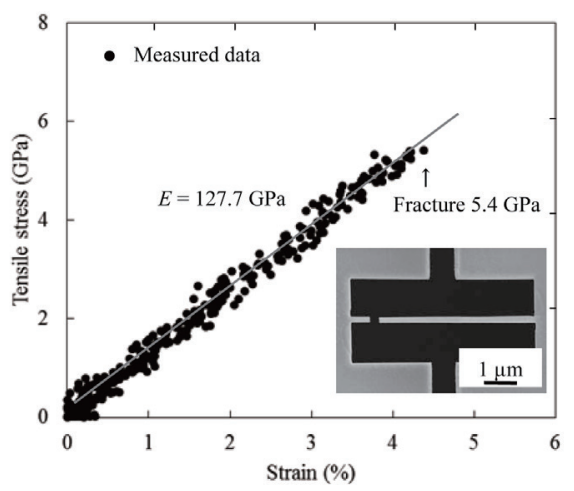

(b)

Fig. 10. (a) SEM image of a Si NW that bridges the gap in the sample stage of device. (b) Representative tensile stress-strain relation of a 163-nm-wide FIB-fabricated Si NW together with an SEM image after failure.

in an FIB system (Hitachi High-Technologies Corp., FB2200). ${ }^{(15-17)}$ The membrane sheet with a Si NW was picked up directly with a W microprobe and transferred to the sample stage in the device. After alignment, W films were deposited to bond the sheet to the stage, as shown in Fig. 10(a). The cross-sectional shape of the NW is almost rectangular. The FIB-fabricated top surface and sidewalls, the thicknesses of which can be estimated to be $90 \mathrm{~nm}$ and $30 \mathrm{~nm}$, respectively, and which have been published elsewhere, were attacked by a Ga ion beam. ${ }^{(15)}$ The affected portion consists of amorphous $\mathrm{Si}$ including $\mathrm{Ga}$, which is known to reduce the mechanical properties of $\mathrm{Si}$ NWs. ${ }^{(15-17)}$ 
Figure 10(b) shows the representative tensile stress-strain relation for a 163 -nm-wide FIBfabricated Si NW together with a SEM image after failure. The strain was calculated by multiplying the measured elongation by a FEA-derived shape factor. ${ }^{(16)}$ The tensile stress-strain relation is almost linear throughout the test until failure, which indicates that the NW fractured in a brittle manner during elastic deformation. The Young's modulus was $127.7 \mathrm{GPa}$, which is $\sim 24.4 \%$ smaller than the bulk value for $\mathrm{Si}(001)[110] .{ }^{(27)}$ The value obtained is in good agreement with our previous results. ${ }^{(16)}$ The fracture strength was $5.4 \mathrm{GPa}$, which is $\sim 11.5$ times higher than that for millimetersized Si structures and poly-crystal Si films. ${ }^{(28,29)}$

\section{Conclusions}

We designed and developed an electrostatically actuated tensile testing system in SEM for Si NWs. The device consisting of electrostatic actuator, capacitive force sensor, and capacitive displacement sensor included a novel stopper mechanism using ETA and ratchet structures to minimize mechanical vibration during specimen test setup. This mechanism improved the yielding percentage by a factor of approximately 5 compared to the devices without the mechanism. It was found in the Type B device that the accuracies of displacement and force measurements were better than $10 \mathrm{~nm}$ and $100 \mathrm{nN}$, respectively, which were enough to conduct nanoscale tensile tests precisely. The FIB-fabricated Si NWs were characterized in an SEM using the device. The Young's modulus and fracture strength of a 163-nm-wide Si NW were 127.7 and $5.4 \mathrm{GPa}$, respectively, which are in good agreement with our previous results.

\section{Acknowledgements}

This work was supported by Grant-in-Aid for JSPS Fellows Grant Number 26.11524.

\section{References}

1 B. Li and C. Lee: Sens. Actuators, A 172 (2011) 61.

2 J. S. Hyun, J.-S. Moon, J.-H. Park, J. W. Kim, Y. D. Kim, and J.-H. Boo: Mater. Sci. Eng. 149 (2008) 292.

3 B. Bhushan: Microelec. Eng. 84 (2007) 387.

4 A. Uranga, J. Verd, E. Marigo, J. Giner, J. L. M-Gamarra, and N. Barniol: Sens. Actuators, A 197 (2013) 88.

5 L. Boodhoo, L. Crudgington, H. M. H. Chong, Y. Tsuchiya, Z. Moktadir, T. Hasegawa, and H. Mizuta: Microelec. Eng. 145 (2015) 66.

6 X. L. Feng, R. He, P. Yang, and M. L. Roukes: Nanolett. 7 (2007) 1953.

7 X. L. Feng, M. H. Matheny, C. A. Zorman, M. Mehregany, and M. L. Roukers: Nanolett. 10 (2010) 2891.

8 M.-H. Park, M. G. Kim, J. Joo, K. Kim, J. Kim, S. Ahn, Y. Cui, and J. Cho: Nanolett. 9 (2011) 3844.

9 H. Wu, G. Chan, J. W. Choi, I. Ryu, Y. Yao, M. T. McDowell, S. W. Lee, A. Jackson, Y. Yang, L. Hu, and Y. Cui: Nat. Nanotechnol. 7 (2012) 310.

10 S. Johansson, J. A. Schweitz, L. Tenerz, and J. Tiren: J. Appl. Phys. 63 (1988) 4799.

11 T. Namazu, Y. Isono, and T. Tanaka: J. Microelectromech. Syst. 9 (2000) 450.

12 T. Ando, M. Shikida, and K. Sato: Sens. Actuators, A 93 (2001) 70.

13 T. Tsuchiya, T. Ikeda, A. Tsunematsu, K. Sugano, and O. Tabata: Sens. Mater. 22 (2010) 1.

14 R. Legtenberg, A. W. Groeneveld, and M. Elwenspoek: J. Micromech. Microeng. 6 (1996) 320.

15 T. Fujii, K. Sudoh, S. Sakakihara, M. Naito, S. Inoue, and T. Namazu: Jpn. J. Appl. Phys. 52 (2013) 110118.

16 T. Fujii, T. Namazu, K. Sudoh, S. Sakakihara, and S. Inoue: J. Eng. Mater. Technol. 135 (2013) 041002.

17 T. Fujii, T. Kozeki, S. Inoue, and T. Namazu: Proc. Solid-State Sens., Act. \& Microsyst., (IEEE, New York, 2015) pp. 900-903. 
18 Y. Zhang, X. Liu, C. Ru, Y. L. Zhang, L. Dong, and Y. Sun: J. Microelectromech. Syst. 20 (2011) 959.

19 D. Zhang, J.-M. Breguet, R. Clavel, V. Sivakov, S. Christiansen, and J. Michler: J. Microelectromech. Syst. 19 (2010) 663.

20 K. T. A. L. Burm: Contrib. Plasma Phys. 47 (2007) 177.

21 J. D. Cobine: Gaseous conductors (Dover, New York, 1958).

22 L. A. Rocha, E. Cretu, and R. F. Wolffenbuttel: J. Microelectromech. Syst. 15 (2006) 69.

23 J. Wu, S. Wang, and J. Miao: J. Microelectromech. Syst. 17 (2008) 921.

24 H. D. Espinosa, Y. Zhu, and N. Moldovan: J. Microelectromech. Syst. 16 (2007) 1219.

25 M. D. Koning, A. Antonelli, M. Z. Bazant, E. Kaxiras, and J. F. Justo: Proc. MRS 539 (1998) 175.

26 Y. Fukuta, H. Fujita, and H. Toshiyoshi: Jpn. J. Appl. Phys. 42 (2003) 3690.

27 H. J. McSkimin: J. Appl. Phys. 24 (1953) 988.

28 H. Kapels, R. Aigner, and J. Binder: IEEE Trans. Electron Devices 47 (2000) 1522.

29 T. Tsuchiya, M. Hirata, N. Chiba, R. Udo, Y. Yoshitomi, T. Ando, K. Sato, K. Takashima, Y. Higo, Y. Saotome, H. Ogawa, and K. Ozaki: J. Microelectromech. Syst. 15 (2005) 1178. 